\title{
Noise reduction efforts for the ALS infrared beamlines
}

\author{
Tom Scarvie, Nord Andresen, Ken Baptiste, John Byrd, Mike Chin, \\ Michael C. Martin, Wayne R. McKinney, Christoph Steier \\ Advanced Light Source Division, Lawrence Berkeley National Laboratory, Berkeley, CA 94720
}

\begin{abstract}
The quality of infrared microscopy and spectroscopy data collected at synchrotron based sources is strongly dependent on signal-to-noise. We have successfully identified and suppressed several noise sources affecting Beamlines 1.4.2, 1.4.3, and 1.4.4 at the Advanced Light Source (ALS), resulting in a significant increase in the quality of FTIR spectra obtained. In this paper, we present our methods of noise source analysis, the negative effect of noise on the infrared beam quality, and the techniques used to reduce the noise. These include reducing the phase noise in the storage ring radio-frequency $(\mathrm{RF})$ system, installing an active mirror feedback system, analyzing and changing physical mounts to better isolate portions of the beamline optics from low-frequency environmental noise, and modifying the input signals to the main ALS RF system. We also discuss the relationship between electron beam energy oscillations at a point of dispersion and infrared beamline noise.
\end{abstract}

\section{CORRESPONDING AUTHOR}

\section{Tom Scarvie}

Advanced Light Source, Ernest Orlando Lawrence Berkeley National Laboratory

1 Cyclotron Rd., MS:80R0114, Berkeley, CA, 94720

Email: tom_scarvie@lbl.gov_ Phone: (510) 487-7697 
KEYWORDS: infrared beamline noise, infrared signal-to-noise, RF phase noise, vibration

\section{INTRODUCTION}

Noise reduction for the infrared beamlines has been an ongoing project since the first ALS beamline was commissioned in 1997 [1-3]. Transverse motions of the photon beam are transformed into intensity variations in the IR signal by an aperture or apertures in the beamline optics or detectors. There are two main sources of this photon beam motion: position and/or energy oscillations of the electron beam at a point of dispersion in the ring (the bending magnet IR source), and mechanical vibrations of beamline components. Higher-frequency noise caused by electron beam motion was dramatically diminished when a quieter master oscillator was installed in the ALS RF system [4,5]. The noise from mechanical vibrations was reduced by mechanically isolating beamline components from environmental vibrations, and the remaining noise damped with an active mirror feedback system [6]. With these major steps achieved, the plan became to identify the next largest noise source, try to reduce or eliminate it, and move on to the next source, until we reached the point where we could not do any better.

\section{DETAILS}

\section{Historical Improvements}

The first major noise reduction at the infrared beamlines came with the installation of a storage ring master oscillator with much lower phase noise than our previous unit [4]. The electron beam current-dependent synchrotron oscillations, a natural feature in all storage rings, were enhanced by the master oscillator phase noise, particularly towards lower frequencies [5]. Installing the RF synthesizer with the lowest phase noise available at the time (an HP8644) dramatically reduced the noise seen in FTIR spectra, as seen in Figure 1. 
The next efforts were focused on isolating the beamline from lower frequency environmental noise $[3,6]$. The beamline was taken off the main ALS cooling water system and a dedicated chiller installed, as the large circulation pumps for cooling the RF cavities caused very noticeable vibrations in the water-cooled first beamline mirror. The concrete pads supporting the front-end mirror assembly and the switchyard optics were cut away from the surrounding 6" thick floor, and bolted to the more stable 12" slab underneath.

A large improvement came with the installation of an active mirror feedback system [7]. The effect of this system is seen in Figure 2. Two dichroic beamsplitters from Spectra-Tech, together with two Hamamatsu position sensitive optical detectors, measure the visible photon beam position and angle. Dual axis Physik Instrumente tip/tilt PZT mirror holders with $1 \mathrm{~mm}$ thick mirrors are then driven by custom feedback circuitry designed and built by the ALS electronics engineering group. The active system can pin the beam at two positions to approximately one micron thus stabilizing the beam in both position and angle at frequencies from DC up to $\sim 1$ KHz.

The infrared beamlines ran quite successfully after the previously mentioned improvements, especially with the final commissioning of the active feedback system in 2000 . Over time, as the beamline scientists gained expertise in the operation of the beamline and detectors, and the FTIR detectors improved, the capabilities of the beamline were pushed to the point where noise in the FTIR spectra started to become limiting again. The hunt for noise was resumed; we would find any remaining noise sources and try to eliminate them, until satisfied that the remaining noise was a natural consequence of the energy or position oscillations of the electron beam IR source. 


\section{Recent Work}

Fourier transformed time domain signals from the first optical detector in the mirror feedback system and from the FTIR detector signal gave us baseline noise measurements. The spontaneous noise from $0-200 \mathrm{~Hz}$ and $0-25 \mathrm{kHz}$ is shown in Figure 3.

In the low frequency range $(0-200 \mathrm{~Hz})$, previous mechanical isolation efforts identified the M1 and M2 beamline mirrors as the main suspects for vibration induced noise. To further characterize the vibration of these mirrors, a Wilcoxon Research Model F3 Electromagnetic Shaker was clamped to the mirror housing and transfer functions were taken by sweeping the driving frequency from $0 \mathrm{~Hz}$ to $200 \mathrm{~Hz}$, and recording the response of the signal from the first optical detector of the mirror feedback system and from the FTIR bench. Upon seeing numerous correlated vibration peaks indicative of mechanical motion, it was discovered that a tensioning spring had been left out of the M2 mirror assembly. The spring was installed while adding motor drive shafts for external adjustment, which also helped to confine mirror motion. Transfer function measurements following these modifications showed a marked improvement in mirror stability, as seen in Figure 4.

One noise type we have always observed is harmonics of the $60 \mathrm{~Hz}$ AC power-line frequency. The $60 \mathrm{~Hz}$ and $180 \mathrm{~Hz}$ peaks can be seen in Fig. 3. It is unclear whether these lines are due to vibrational modes of the electron beam, or are introduced in the detector equipment itself. In an effort to damp these lines, the RF group installed a low-pass filter on the output of the main RF synthesizer, and also tried an optical link from the synthesizer to the klystron, which should have eliminated the effects of any ground loops within the RF system. Neither of these methods reduced the $60 \mathrm{~Hz}$ lines seen at the IR beamline to any noticeable degree.

At higher frequencies $(500 \mathrm{~Hz}-25 \mathrm{kHz})$, mechanical vibrations are unlikely to be the cause of noise, so any introduced noise is most likely coming from transverse motion of the electron beam, or from energy oscillations of the beam at the bend magnet source, a point of dispersion. 
Figure 3 shows that there are noticeable noise peaks centered at about $7.5 \mathrm{kHz}$ and $18 \mathrm{kHz}$ in the $\mathrm{y}$-axis of the optical detector and in the overall IR signal, but none in the x-axis of the optical detector signal. Due to the configuration of the beamline, $\mathrm{x}$-axis beam motion is observed on the $y$-axis of the detector, and vice-versa, and so the traces correspond to what one would expect for horizontal beam oscillations. We know that the broad $7.5 \mathrm{kHz}$ peak is due to synchrotron oscillations of the beam, because it slowly shifts as the beam current decays and also changes with beam energy. One possibility was that the ALS longitudinal feedback system (LFB) was contributing to this noise by amplifying the synchrotron peak. By comparing the beamline noise with the LFB set to lower and higher gains, we were able to eliminate this as a possibility. This oscillation is a fundamental property of a storage ring source, so we are unsure if there are other ways to damp it. The $18 \mathrm{kHz}$ peak, on the other hand, was also observed on the $\mathrm{x}$-axis signal of a storage ring beam position monitor. This noise was found to be coming from a DAC output in the storage ring RF phase shifter system, which is used in orbit correction. The output of the DAC was filtered by the RF group and Figure 5 shows the result - the $18 \mathrm{kHz}$ noise is almost completely gone.

\section{CONCLUSION}

Noise reduction efforts at the infrared beamlines have been an ongoing process since the commissioning of the beamlines. Upgrading the ALS RF system, and isolating the beamline from environmental noise, eliminated the major noise sources. An active mirror feedback system damped much of the remaining noise below $\sim 1 \mathrm{kHz}$. Recently, a mechanical flaw in a beamline mirror and an electrical problem in the RF system were fixed, and the signal-to-noise in the infrared beam is better than ever. Our plan for further improvement includes more thoroughly examining the beamline optics between the M2 mirror and sample stage to identify any aperture(s) where the photon beam motion is transformed into an amplitude modulation of the IR 
signal. Additionally, a fast orbit feedback system is being commissioned at the ALS that should help quiet transverse beam motions below about $60-70 \mathrm{~Hz}$. It will be interesting to see if this improves the quality of the signal at the infrared beamlines. With further noise improvements, we hope to bring the signal-to-noise ratio to a level competitive with traditional thermal IR sources and increase the demand for synchrotron-based infrared science.

\section{REFERENCES}

1. W. R. McKinney, et al, SPIE Proceedings, 1997, Vol. 3153, pp. 59-76.

2. M. C. Martin and W. R. McKinney, Proceed. Mater. Res. Soc., 524, 11, 1998.

3. W.R. McKinney, M.C. Martin, J.M. Byrd, R. Miller, et al., SPIE Proceedings, 1999, Vol. 3775 , p. 37.

4. J.M. Byrd, M.C. Martin, and W.R. McKinney, Proceedings of the 1999 Particle Accelerator Conference, Editors: A. Luccio and W. MacKay, (New York, 1999), p. 495.

5. J.M. Byrd, Proceedings of the 1999 Particle Accelerator Conference, Editors: A. Luccio, W. MacKay, (New York, 1999), p. 1806.

6. J.M. Byrd, M.Chin, M.C. Martin, W.R. McKinney, and R. Miller, SPIE Proceedings, 1999, Vol. 3775, pp.58-64.

7. A detailed description of the active mirror feedback system is available at http://infrared.als.lbl.gov/feedback. This page also has links to the main ALS Infrared Website, where PDF's of most of these references are available.

This work was supported by the Director, Office of Energy Research, Office of Basic Energy Sciences, Materials Science Division, of the U.S. Department of Energy under Contract No. DEAC03-76SF00098. 


\section{FIGURE CAPTIONS}

Figure 1. Three FRIR spectra showing the effects of the master oscillator (MO) phase noise. The upper two traces were taken with the old MO, the bottom trace with the new MO.

Figure 2. The left figure shows measured noise spectrum at the first Hamamatsu detector with and without one feedback loop on. The largest vibration at $80 \mathrm{~Hz}$ is reduced by a factor of 15 . The right figure shows variation of the interferogram zero burst height as a function of time with the feedback system on and off.

Figure 3. Spontaneous noise spectra at the Infrared Beamline, prior to any of the recent improvements discussed in this report.

Figure 4. Transfer functions of the IR signal before and after the M2 mechanical modification. A distinct slope in the lower phase plot indicates a vibration correlated to the driving frequency and therefore a significant mechanical oscillation. Many of the mechanical vibration modes were reduced, especially those at $65 \mathrm{~Hz}, 110 \mathrm{~Hz}, 128 \mathrm{~Hz}$. The peak that arose at $175 \mathrm{~Hz}$ is still under investigation.

Figure 5. 0-25 kHz spontaneous noise on the first mirror feedback optical detector before and after RF phase shifter DAC filtering. The upper and lower plots represent vertical and horizontal electron beam motion, respectively. Different scopes were used, but the ranges are the same. Notice the substantial reduction in the $18 \mathrm{kHz}$ peak in the bottom-right plot. The broad peak around $7.5 \mathrm{KHz}$ is due to synchrotron oscillations. 
Figure 1:

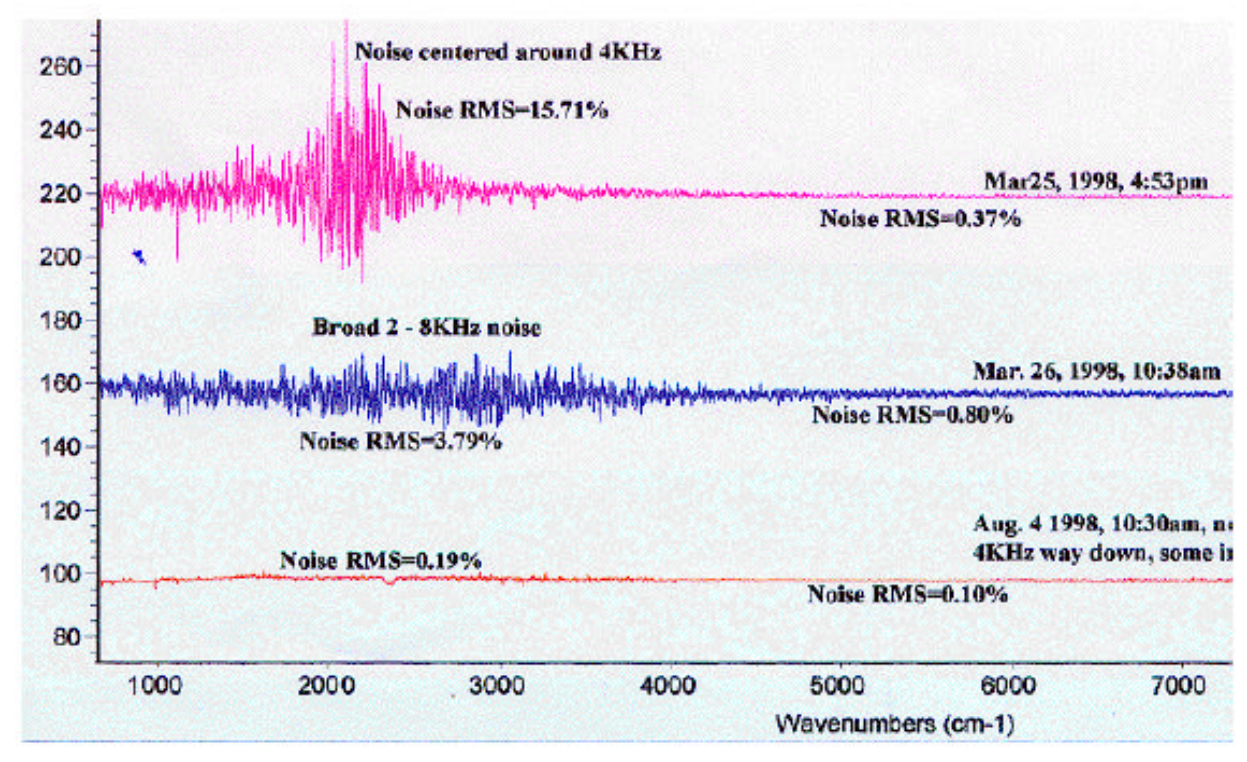

Figure 2:
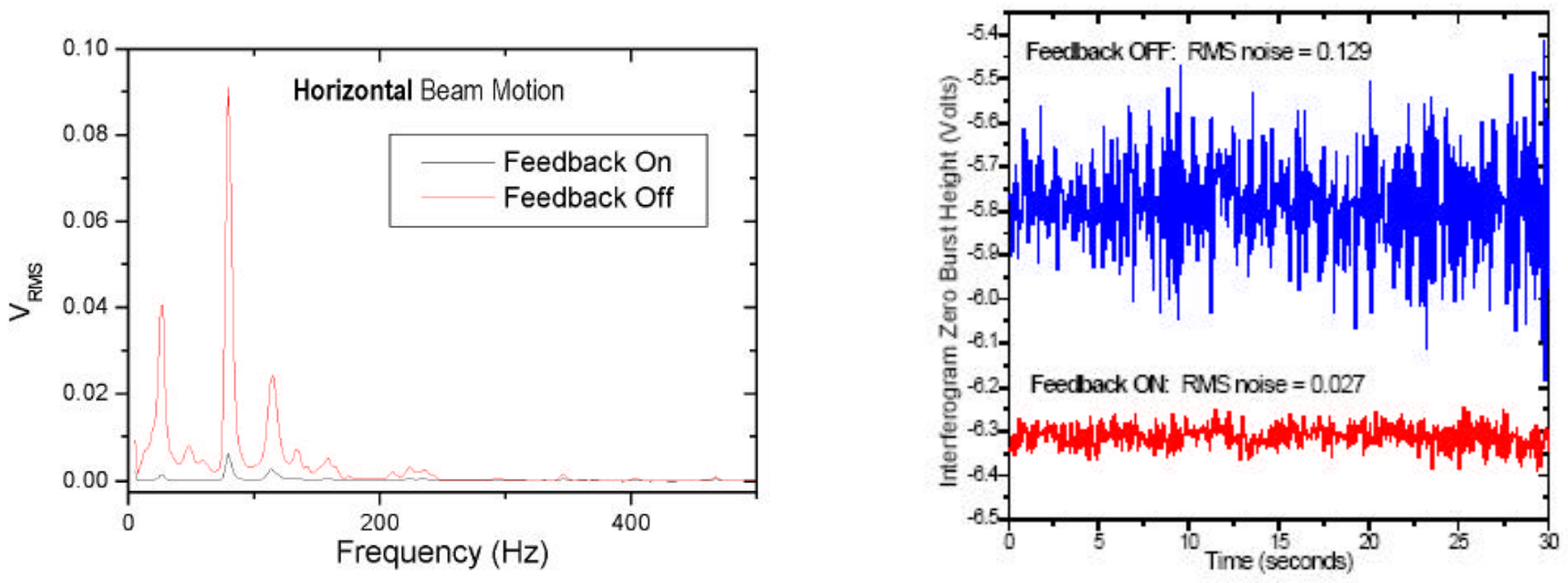
Figure 3:

TRACE A: Ch2 Spectrull

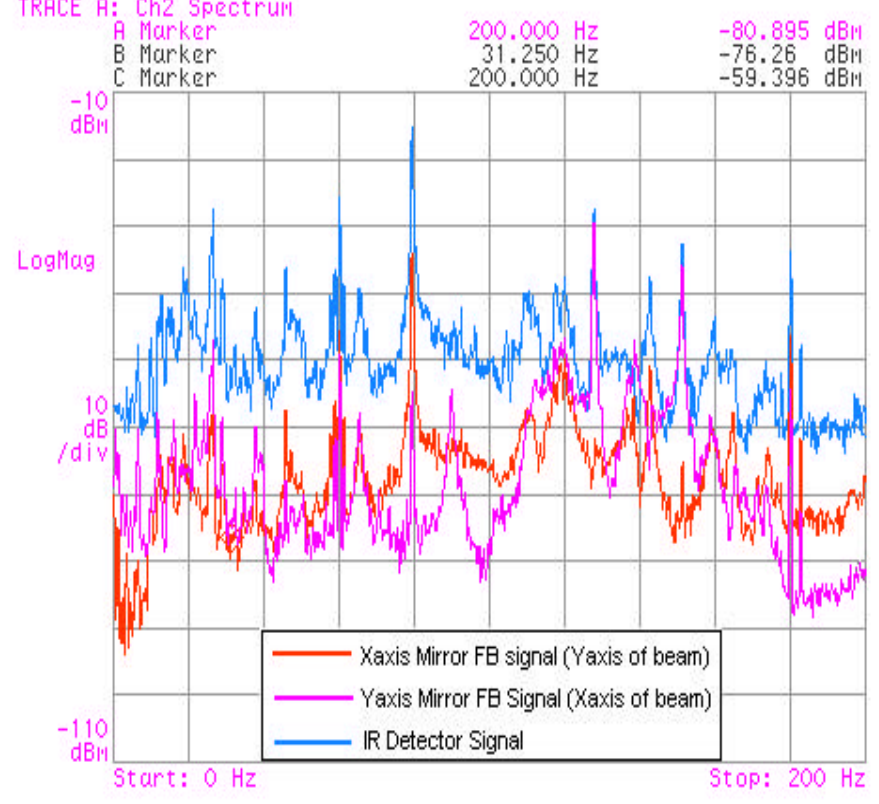

TRACE A: Ch2 Spectrull

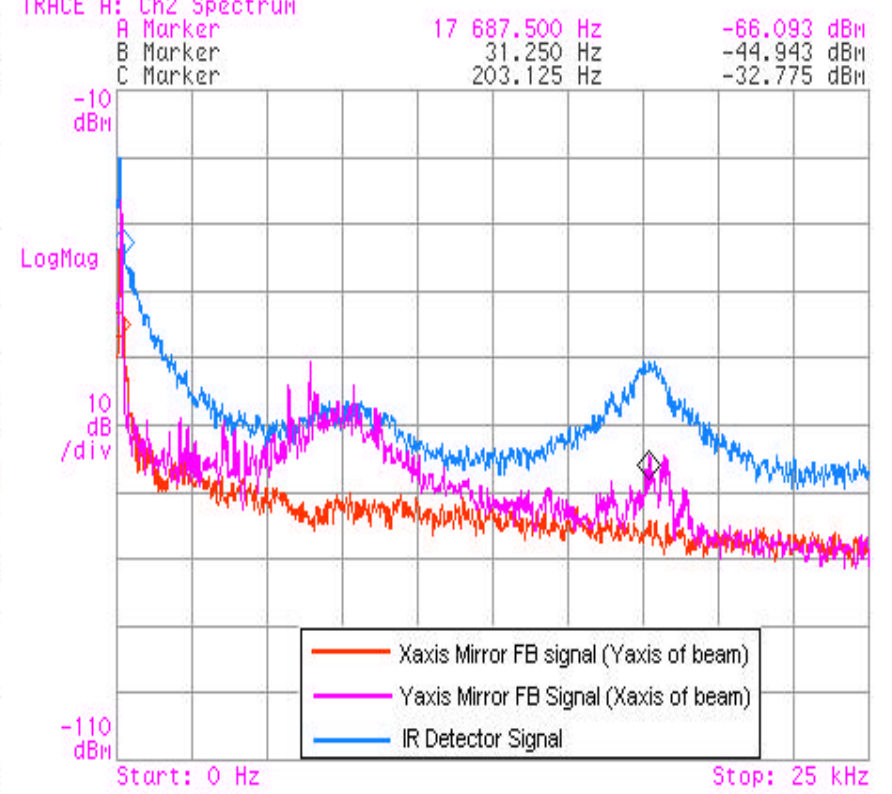


Alternate Figure 3 (better for black and white):

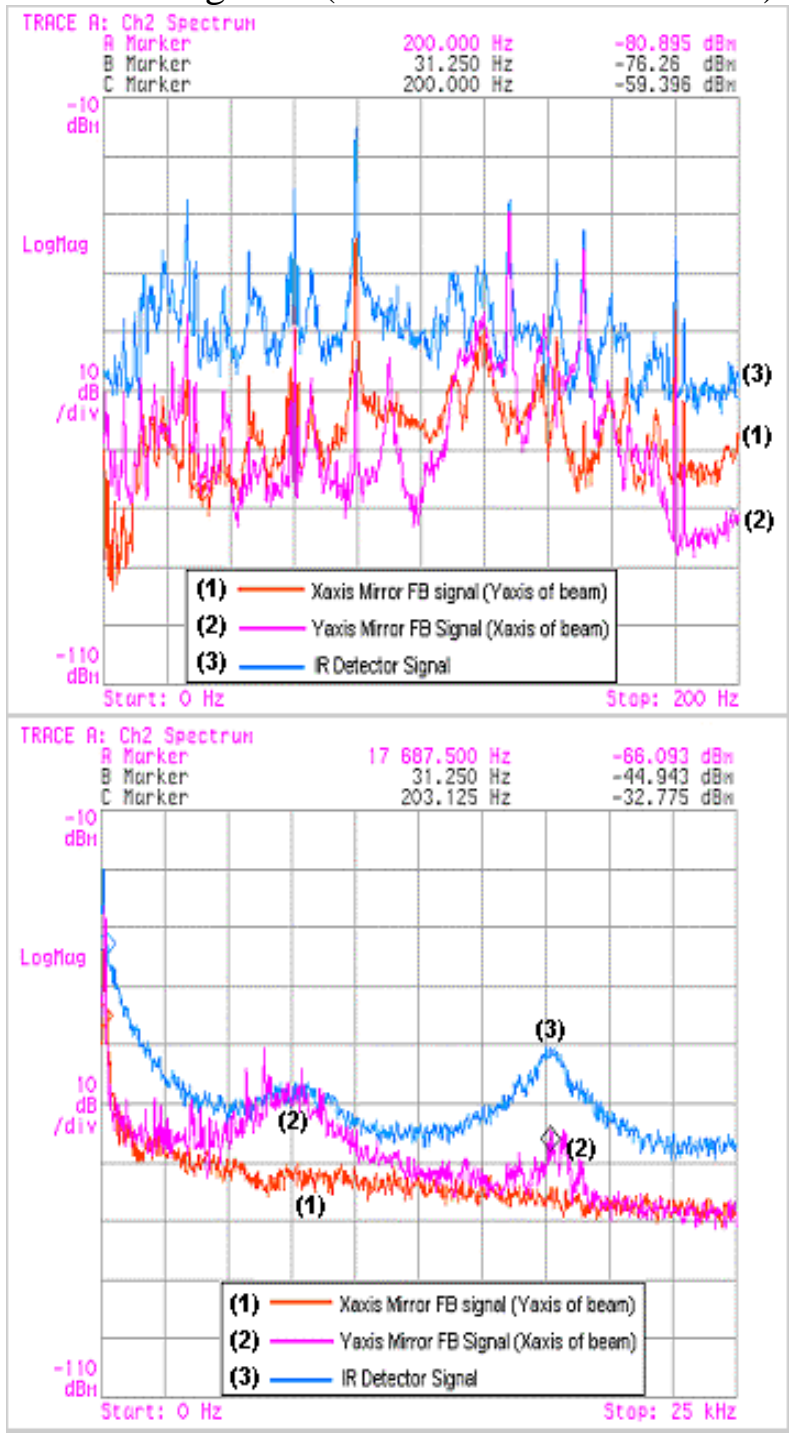

Figure 4:
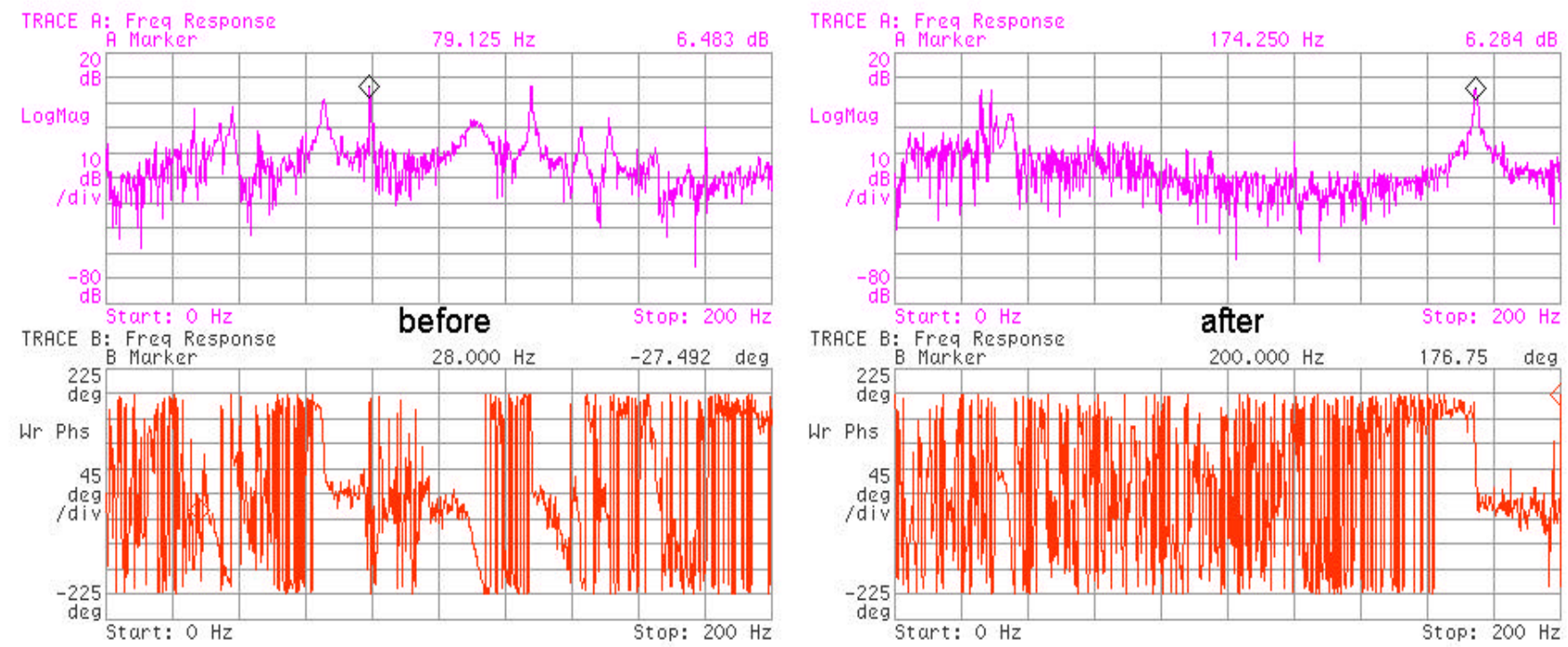
Figure 5:
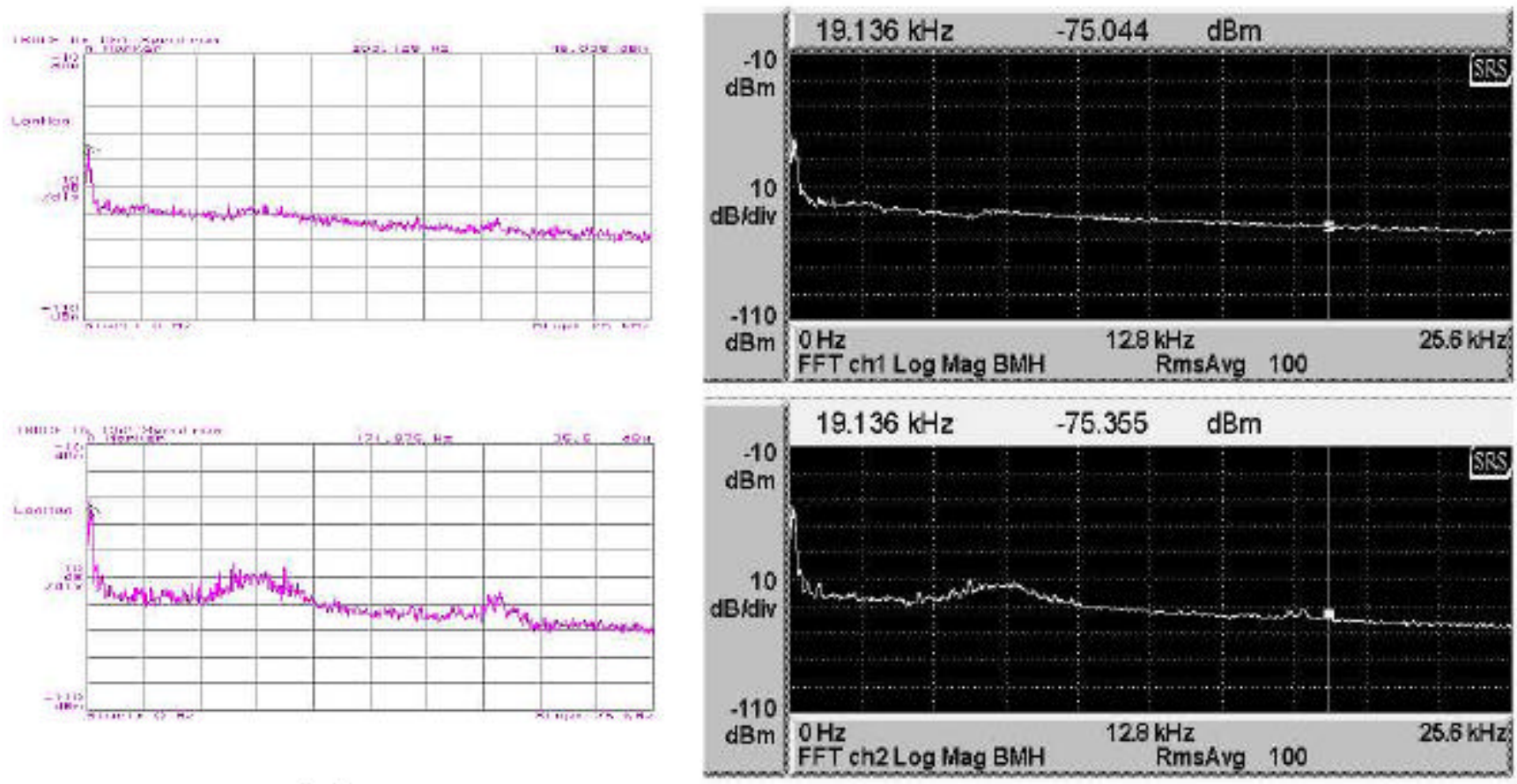

before 